\title{
Limits of flow-cytometry histogram analysis methods to assess bladder tumour antigen expression
}

\author{
Agnès Chabanas ${ }^{1}$, Jean-Jacques Rambeaud ${ }^{2}$, Hong-Hsu Huo ${ }^{2}$, Daniel Seigneurin ${ }^{3}$ and \\ Jean-Jacques Lawrence ${ }^{1, *}$ \\ ${ }^{1}$ Laboratoire de Biologie Moléculaire du Cycle Cellulaire, INSERM U309, \\ ${ }^{2}$ Service d'Urologie, Centre Hospitalier Universitaire de Grenoble, \\ ${ }^{3}$ Bases Moléculaires de la Progression Tumorale, \\ ${ }^{1,3}$ Institut Albert Bonniot, Université Joseph Fourier-Grenoble I, France
}

\begin{abstract}
Tumour-associated antigens detected in cells obtained from bladder washings or tumours are useful markers in bladder cancer. Flow cytometry is commonly used to quantify immuno-stained cells. A straightforward way to analyze data is to count the fluorescent cells above a threshold empirically determined on a control histogram representation. However, specific antigens expressed at highly variable rates give rise to wide range distributions in flow cytometry as illustrated when a mucin antigen for urinary bladder was titrated by M344 monoclonal antibody in urothelial cancer cells. We have evaluated several methods of background estimation and subtraction in order to determine the proportion of M344 Mab positive cells. These include threshold setting (Histogram Shape Dependent (HSD) threshold developped in this study, 2\% preset or 5\% preset background), subtraction of the blank from the test histograms, and Kolmogorov-Smirnov statistical test. The HSD method appeared to be a more reliable method for background estimation; however, in the case of very low antigen expression, where specific fluorescence histograms could hardly be distinguished from that of the background, fluorescence microscopy remained the only valid method, since it allowed the distinction between specific and non-specific fluorescence on the basis of structural differences between the two.
\end{abstract}

Keywords: Tumour antigens, flow cytometry, histogram analysis, bladder cancer

\section{Introduction}

A number of tumour antigens, defined by monoclonal antibodies (Mab), are markers that may provide information useful for the diagnosis, follow-up and possibly prognosis in bladder cancer (reviews in $[1,4,6]$ ). Flow cytometry is commonly used to detect and quantify cells from bladder irrigations or tumours reactive with Mabs.

The simplest and the most often used method of flow cytometry data analysis to assess the reactivity of cells with Mabs, in clinical samples, is to determine the number of cells positively stained as those

${ }^{*}$ Corresponding author: Jean-Jacques Lawrence, INSERM U309, Institut Albert Bonniot, Université Joseph Fourier Grenoble I, Domaine de la Merci, 38706 La Tronche Cedex, France. Tel.: +33 4765495 77; Fax: +33 4765495 95, E-mail: jean-jacques.lawrence@ujf-grenoble.fr. 
having fluorescence intensities above a threshold level of fluorescence empirically determined from a control sample. The threshold may also be fixed by giving a preset background of $2 \%$, or $5 \%$, of the control cells, respectively [11,12]. These methods are usually valid when there is a clear difference between the fluorescence intensities of sample and control cells, i.e., when histograms do not overlap.

However, tumour antigens in bladder tumours are often expressed at variable levels and, in many cases, the fluorescence distributions of the test and control cells frankly overlapped; in such cases the immunofluorescence intensities of a significant fraction of sample cells might be similar to that of non-specific immunofluorescence of control cells, although they are different in nature. The limit of fluorescence threshold is thus questionable. We have encountered samples for which the intensity of fluorescence in immunofluorescent cells was very low and these cells would have been classified as negative by conventional criteria. However, a rapid fluorescence microscopy examination of such samples revealed that these cells were weakly, but specifically, stained as revealed by the structure of the fluorescent pictures. It appeared then, that it was essential to test the limits of validity of the histogram analysis method, especially when antigens are weakly expressed in the cells.

We propose an improved threshold determination, taking into account the shapes of the distribution curves of immunofluorescence, named histogram shape dependent threshold (HSD) and show that this method may lead to valuable results in cases where the other methods gave contradictory results.

We have compared results obtained by the existing threshold method with those obtained from the same data by other analytical methods. First, thresholds were fixed on the control histograms selecting preset proportions of the most stained cells: $2 \%$ or $5 \%$ of the cell samples $[11,12]$. Second, another method consisted of subtracting the control histogram from the test histogram, on a channel by channel basis $[12,13]$. Third, a statistical method was essayed to test the probability of a difference between the control and test fluorescence distributions [5,15]. A direct microscopic examination was used to test the validity of the results obtained with any of the above histogram analysis methods.

For this study, we analyzed the expression of the bladder cancer mucinous antigen, an early marker of urothelial transformation, revealed by the M344 monoclonal antibody. This antigen is expressed in $60 \%$ of $\mathrm{Ta} / \mathrm{T} 1$ bladder tumours, as revealed by immunohistology methods on tissue sections [7]; moreover, the cellular immunofluorescence pattern is typically granular, with a frequent polarity, and the identification of positive cells in fluorescence microscopy is unquestionable [8]. This antigen was considered to be a good model since it presents a variable level of expression in bladder tumour cells.

Since non-urothelial cells (stromal, inflammatory or blood cells) were present in variable amounts in tumours or in washing samples, the urothelial cells, reactive for the pan-urothelial T16 Mab [9], were quantified. This had two advantages: the urothelial antigen expression was used as an internal reference to assess the M344 positivity of the samples, minimizing inter-sample variations; moreover, the T16 antigen being strongly expressed, its expression served as a reference to compare results of the different data analysis methods for highly fluorescent cells.

\section{Materials and methods}

\subsection{Sample collection and preparation}

Seven bladder washing specimens and 12 tumours were obtained from 15 patients with superficial tumours (Ta, T1).

Bladder irrigations were performed, before resection, by ten vigorous bladder washes made through a rectoscope $25 \mathrm{~F}$, using 200 to $300 \mathrm{ml}$ saline solution. Specimens were centrifuged at $200 \mathrm{~g}$ for $10 \mathrm{~min}$ 
and cells were resuspended in phosphate buffered saline solution without calcium and magnesium (PBS). Aliquots of $2 \times 10^{6}$ to $4 \times 10^{6}$ urothelial cells were suspended in $1 \mathrm{ml}$ freezing medium composed of RPMI (Boehringer Mannheim, Ger.), 15\% fetal calf serum (FCS) and 10\% dimethyl sulfoxide; they were frozen at $-80^{\circ} \mathrm{C}$ and stored in liquid nitrogen.

Tumour specimens were obtained using a section mode without coagulation. Tumour fragments were mechanically dissociated in PBS and, after two washes, cells were frozen as described above.

\subsection{Immuno-staining}

After thawing at $37^{\circ} \mathrm{C}$ in $10 \mathrm{ml}$ of medium composed of RPMI and $15 \% \mathrm{FCS}$, cells were collected by centrifugation (200 g, $10 \mathrm{~min}$ ) and washed once in PBS. As described previously [3], separate aliquots were stained for the antigenic expression.

Tumour antigens were immunostained using the M344 monoclonal antibody (Mab); urothelial cells were specifically stained using the T16 Mab and the SP2/0-Ag14 myeloma culture supernatant was used as a negative control. $2 \times 10^{5}$ to $1 \times 10^{6}$ urothelial cells were incubated for $1 \mathrm{~h}$ at $4^{\circ} \mathrm{C}$ in $100 \mu \mathrm{l}$ of undiluted hybridoma (or SP2/0) culture supernatant, washed twice in cold PBS- $0.5 \%$ bovine serum albumin (BSA) and incubated for $30 \mathrm{~min}$ at $4^{\circ} \mathrm{C}$ with $100 \mu \mathrm{l}$ of fluorescein-conjugated $\left.\mathrm{F}(\mathrm{ab})\right)^{\prime 2}$ fragment goat anti-mouse IgG solution (1:100 working dilution in PBS-2\% BSA) (Jackson Immuno Research Lab., PA). After one wash, cells were resuspended in $1 \mathrm{ml} \mathrm{PBS}$ and fixed by slowly pouring the cell suspension into $1 \mathrm{ml}$ of ethanol, for one hour at room temperature.

\subsection{Flow-cytometry analysis}

The stained cells were analyzed on a FACStar Plus, as described in [3]; the fluorescence intensities were collected in a list mode and the data were analyzed with the Lysis II Management System (Becton Dickinson). After gating to exclude debris and doublets, 7,000 to 9,000 cells for each sample were analyzed simultaneously for antigen.

\subsection{Methods of data analysis for tumour antigen expressions}

Several methods were used to evaluate the percentage of positive cells; they all required negative control (blank) and test histograms.

Variants of the threshold method. As shown in Fig. 1, one threshold value of fluorescence, taking into account the shape of the distribution curve, was visually fixed on the right of the blank histogram, approximately at the intersection point of the curve slope with the x-axis; the cells with an autofluorescence higher than the threshold defined a variable background, named Histogram Shape Dependent background (HSD). Two other thresholds were fixed in a such way that they selected a preset background equal to $2 \%$ and $5 \%$ of the control cells.

For each threshold method, the percentages of M344 and T16 fluorescent cells were calculated by integrating the test histogram on the right of the threshold. To avoid false positives, the percentage of positive cells was estimated by subtracting the percentage of background from the percentage of fluorescent cells [12,14].

The reactivity of a sample with M344 Mab was determined, as proposed by Fradet et al. [9], as the ratio of the percentage of M344 positive cells to the percentage of T16-stained urothelial cells; positive samples having a ratio $>0.1$. 


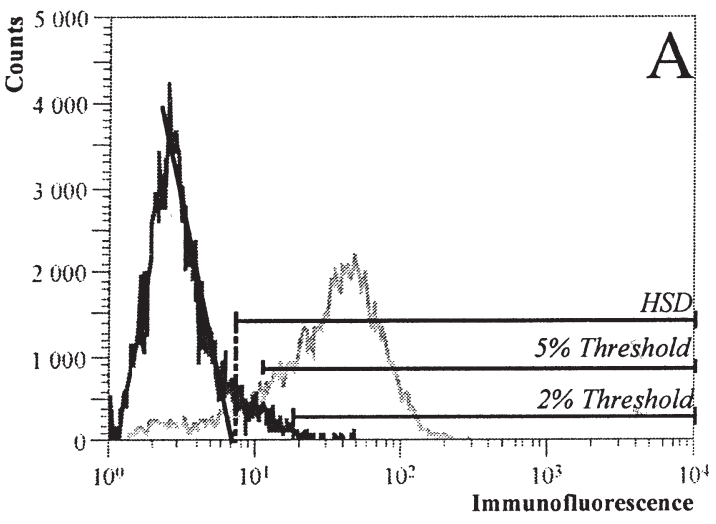

Tumours

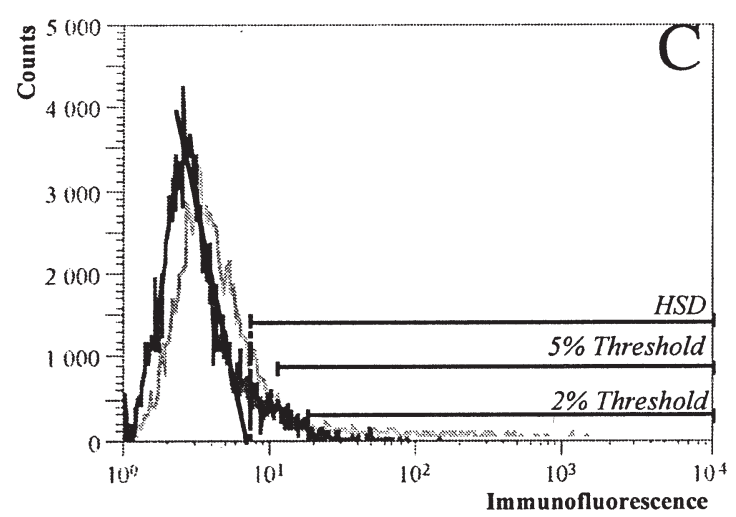

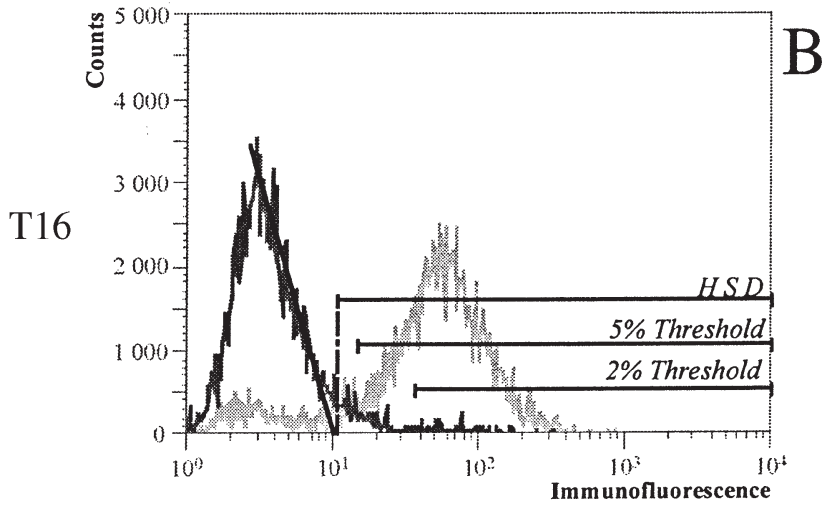

Washings

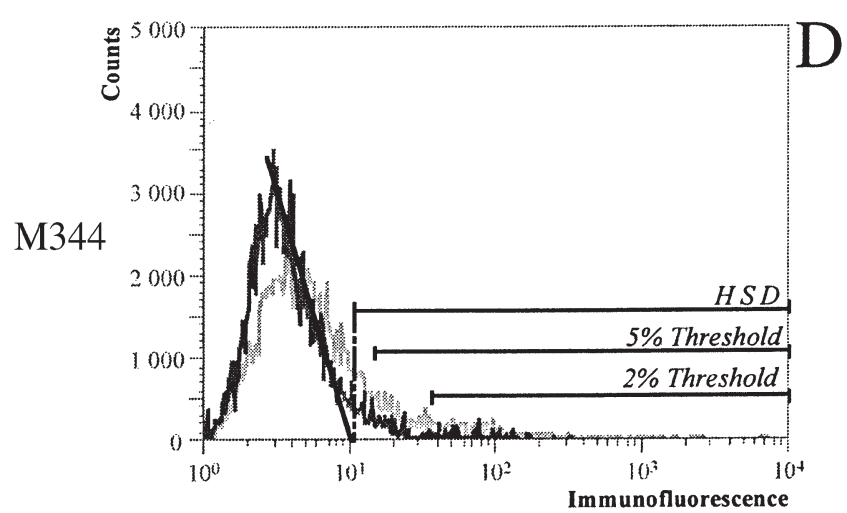

Fig. 1. Threshold settings from blank histograms. The immunofluorescence of control cells is represented in black histograms and that of sample cells in grey histograms, for T16 antigen expression (A and B) and M344 antigen expression (C and D), in either tumour cells (A and C) or cells from bladder washings (B and D). The settings of the threshold used to determine the number of positive cells in sample histograms is indicated by the horizontal bars in each histogram and was determined from the corresponding control histogram (HSD stands for Histogram Shape Dependent threshold).

The subtraction method. Using the Histogram Tools option of the Lysis II program, we subtracted, channel by channel, the cell counts of the blank histogram from the cell counts of the test histogram. The positive percentage was determined as the ratio of the sum of all positive differences to the total test count.

A statistical method was used to assess the positivity of the samples without calculating the proportion of positive cells; with the non-parametric Kolmogorov-Smirnov (K-S) Two-Sample test [15], available in the same option of the computer program, we calculated the probability of a significant difference between the frequency distributions of the test and control cells.

\subsection{Control by fluorescence microscopy}

After flow-cytometry analysis, aliquots of remaining cell suspensions of four samples were cytocentrifuged (100 g, $5 \mathrm{~min}$ ). Slides were mounted with an anti-fading (DABCO, Sigma, F). The cells specifically stained for antigen expression and the negative cells were counted with an optical microscope. 


\section{Results}

Cells of seven bladder washing specimens and 12 tumours were analyzed by flow cytometry for M344 tumour antigen expression and for T16 urothelial cell surface antigen expression. For four patients, irrigations and tumours were obtained concomitantly.

To assess the tumour antigen expression in a sample, the final question for clinicians, the proportion of M344-positive cells was reported to the proportion of T16-positive urothelial cells, since the proportion of urothelial cells varies from one sample to another [9]. The proportion of cells expressing these antigens must then be determined accurately.

\subsection{Background evaluation}

Three different fluorescence threshold values were defined on the control histograms, as described in Section 2, determining three background values (Fig. 1): HSD, 2\% preset and 5\% preset. The HSD backgrounds ranged from $2 \%$ to $5 \%$ of the cells for tumours and from $4 \%$ to $8 \%$ for washings, except for one patient for whom $20 \%$ of the washing cells displayed an intense non-specific fluorescence.

\subsection{Urothelial cell estimation by T16 expression}

The differentiation antigen T16 was strongly expressed in all the cases, as illustrated in Fig. 1: most cells were highly fluorescent and the fluorescence histograms were clearly distinct from the blank histograms.

As expected, the average percentages of T16 fluorescent cells in tumours did not depend on the method used for threshold determination (Table 1); at least $70 \%$ of urothelial cells were assessed in all tumour samples. In washing samples, blood cells were frequently present and the non-specific fluorescence of urothelial cells was usually greater than for cells obtained directly from tumours (Fig. 1). Therefore, the proportions of fluorescent cells were not as high in washing samples as in tumours. The differences were largest when the evaluations were made using a preset $2 \%$ threshold.

Nevertheless, the percentages of urothelial cells remained high enough, representing at least $50 \%$ of the total cells analyzed, and accordingly T16 urothelial cell expression was considered as a suitable internal reference marker for cells originating from both bladder washings and tumour samples.

Table 1

Expression of the T16 differentiation antigen and of the M344 tumoral antigen on cells of bladder washing and tumour samples, studied by flow cytometry analysis of Mab stained cells. The percentages of fluorescent cells were calculated from three different thresholds of fluorescence, fixed on the blank histograms, determining different background values. The Histogram Shape Dependent backgrounds range from $2 \%$ to $5 \%$ of the cells for tumours and from $4 \%$ to $8 \%$ for all the washes but one

\begin{tabular}{|c|c|c|c|c|c|c|c|}
\hline \multirow[t]{2}{*}{ Antigens } & \multirow[t]{2}{*}{ Samples } & \multicolumn{2}{|c|}{ HSD } & \multicolumn{2}{|c|}{$\begin{array}{c}\text { Background method } \\
2 \% \text { Preset }\end{array}$} & \multicolumn{2}{|c|}{$5 \%$ Preset } \\
\hline & & $\%$ (Mean) & Range (\%) & $\%$ (Mean) & Range & $\%$ (Mean) & Range \\
\hline T16 & $\begin{array}{l}\text { Tumours (12) } \\
\text { Washings (7) }\end{array}$ & $\begin{array}{l}87 \\
81\end{array}$ & $\begin{array}{l}77 \text { to } 99 \\
68 \text { to } 91\end{array}$ & $\begin{array}{l}86 \\
68\end{array}$ & $\begin{array}{l}70 \text { to } 99 \\
48 \text { to } 87\end{array}$ & $\begin{array}{l}89 \\
81\end{array}$ & $\begin{array}{l}77 \text { to } 99 \\
65 \text { to } 91\end{array}$ \\
\hline M344 & $\begin{array}{l}\text { Tumours (12) } \\
\text { Washings (7) }\end{array}$ & $\begin{array}{l}36 \\
17\end{array}$ & $\begin{array}{l}4 \text { to } 78 \\
7 \text { to } 40\end{array}$ & $\begin{array}{c}33 \\
9\end{array}$ & $\begin{array}{l}2 \text { to } 66 \\
2 \text { to } 29\end{array}$ & $\begin{array}{l}42 \\
16\end{array}$ & $\begin{array}{l}4 \text { to } 78 \\
6 \text { to } 40\end{array}$ \\
\hline
\end{tabular}


Table 2

M344 tumour antigen expression in bladder washings and tumour samples. For each background value (Histogram Shape Dependent, 2\% or 5\%), sample reactivities were determined as the ratio of \% M344 positive cells over \% T16 urothelial cells; positive samples have a ratio $>0.1$ [8]. The table give the number of samples which are positive $(+)$ or negative $(-)$ with the three thresholds of fluorescence. "Variable" samples have results that differ according to the threshold method used (examples: washing of patient 1 and tumour of patient 2, in Table 3)

\begin{tabular}{lccc}
\hline Samples & \multicolumn{3}{c}{ M344 reactivity } \\
\cline { 2 - 4 } & + & - & "Variable" \\
\hline Tumours (12) & 9 & 1 & 2 \\
Washings (7) & 3 & 2 & 2 \\
\hline
\end{tabular}

\subsection{Tumour cell determination by M344 expression}

The tumour M344 antigen was expressed at highly variable degrees in a given sample, as well as from one sample to another. Some samples had a large amount of highly fluorescent cells; in many other cases, the majority of fluorescent cells were weakly stained, and, only a small cell fraction was strongly stained. All samples had a large distribution of fluorescence intensities and in all instances there was a marked overlap of the test and control histograms (examples in Fig. 1). It has to be emphasized that these observations neither depended on the fixation nor on the staining procedures utilized. Several protocols were tested, including those which involve a fixation with ethanol before the staining procedure, all giving essentially the same results.

A smaller fraction of cells was more reactive with the M344 than with the T16 Mabs, in washings as well as in tumours (Table 1). However, differences were observed according to the mode of sample collection: the percentages of M344 positive cells were lower in irrigation fluids than in tumours. Differences also appeared to depend on the method used to evaluate the background of fluorescence. The proportion of fluorescent cells was frequently lower when assessed with a $2 \%$ preset background than by the two other methods of threshold setting.

As a consequence of the variable estimations of the percent of fluorescent cells, the classification of tumour and washing samples as positive or negative for M344 expression might be questionable. As shown in Table 2, 12 samples out of 19 were positive for M344 expression whichever the threshold of fluorescence used, and three samples were negative. However, in four cases results depended on the threshold setting method.

\subsection{Comparison of positive cell number evaluation methods}

As we found that the positivity of a sample for M344 expression could depend on the threshold setting method utilized, we attempted to use two other tools available on commercial instruments for histogram analysis and background evaluation.

Subtraction method. This method was used to reprocess the flow-cytometry data for the seven negative or "variable" samples; the control histogram was subtracted from the test histogram; the remaining cells, were considered as positive cells. As expected, the percentage of T16 positive cells (mean 80\%, range 72 to $89 \%$ ) did not differ significantly from the percentage of urothelial cells calculated by the threshold methods. 
Table 3

Percentage of M344 positive cells assessed by each histogram analytical method and corresponding sample reactivities

\begin{tabular}{|c|c|c|c|c|c|c|c|c|c|c|c|}
\hline \multirow{4}{*}{ Patient } & \multirow{4}{*}{ Sample } & \multicolumn{8}{|c|}{ Histogram analysis method } & \multirow{3}{*}{\multicolumn{2}{|c|}{$\begin{array}{c}\text { Fluorescence } \\
\text { microscopy }\end{array}$}} \\
\hline & & \multicolumn{6}{|c|}{ Background evaluation } & \multirow{3}{*}{\multicolumn{2}{|c|}{$\begin{array}{l}\text { Blank subtraction } \\
\text { Cells }+ \text { Reactivity }\end{array}$}} & & \\
\hline & & & HSD & $2 \%$ & Preset & $5 \%$ & Preset & & & & \\
\hline & & Cells + & Reactivity & Cells + & Reactivity & Cells+ & Reactivity & & & Cells & Reactivity \\
\hline \multirow[t]{2}{*}{1} & Washing & $10 \%$ & $\stackrel{+}{+}(0.12)$ & $4 \%$ & $(0.06)$ & $9 \%$ & $\stackrel{+}{+}$ & $24 \%$ & $\begin{array}{c}+ \\
(0.31)\end{array}$ & $10 \%$ & $\begin{array}{c}+ \\
(0.13)\end{array}$ \\
\hline & Tumour & $25 \%$ & $\begin{array}{c}+ \\
(0.28)\end{array}$ & $17 \%$ & $\stackrel{+}{+}$ & $25 \%$ & $\begin{array}{c}+ \\
(0.28)\end{array}$ & $36 \%$ & $\begin{array}{c}+ \\
(0.40)\end{array}$ & $21 \%$ & $\begin{array}{c}+ \\
(0.26)\end{array}$ \\
\hline 2 & Tumour & $9 \%$ & $\begin{array}{l}-? \\
(0.1)\end{array}$ & $5 \%$ & $(0.06)$ & $18 \%$ & $\stackrel{+}{+}$ & $31 \%$ & $\begin{array}{c}+ \\
(0.35)\end{array}$ & $7 \%$ & $(0.08)$ \\
\hline 3 & Tumour & $19 \%$ & $\stackrel{+}{+}$ & $19 \%$ & $\stackrel{+}{+}$ & $33 \%$ & $\stackrel{+}{+}$ & $43 \%$ & $\begin{array}{c}+ \\
(0.44)\end{array}$ & $21 \%$ & $\begin{array}{c}+ \\
(0.21)\end{array}$ \\
\hline
\end{tabular}

${ }^{*}(\% \mathrm{M} 344$ cells $+/ \%$ T16 urothelial cells +$)$.

All samples could be reclassified as positive for the M344 antigen: as shown in Table 3, third column, they had a proportion of positive cells higher than those assessed by the three threshold methods; the ratio $\% \mathrm{M} 344^{+}$over $\% \mathrm{~T}^{+} 6^{+}$was clearly higher than the limit of positivity for these samples.

Statistical analysis. Using the K-S test, we calculated the probability of a statistical difference between the test and control frequency distributions for the seven samples considered as "variable" or negative with the threshold methods. As with the subtraction method, all samples were reclassified as positive: the fluorescence distributions of the test cells were more or less shifted to higher fluorescence values and the blank and test distributions differ significantly ( $99 \%$ probability of difference). However, again as with the subtraction method, the risk of false positivity was demonstrated: the tumour of patient 2, classified as positive by this method, appeared negative by fluorescence microscopy (see below).

\subsection{Control by fluorescence microscopy and comparison of the different methods}

Four cell suspensions stained by M344 or T16 Mabs were cytocentrifuged and positive and negative cells were counted through a fluorescence microscope. At least 250 cells were examined for each sample. T16 or M344 negative cells had a yellowish or orange coloured uniform fluorescence, as negative controls. The T16 dotted pattern of fluorescence was very regular. Percentages of T16 positive urothelial cells were similar to those determined by the threshold methods.

The pattern of fluorescence after M344 staining was much more heterogeneous, with a number of green grains ranging from one to high, or uncountable. Percentages of specifically fluorescent cells and sample reactivities are reported in Table 3 (last column). It appears that, for these four samples, the percentages of specifically-stained cells assessed by microscopy were much lower than the percentages of positive cells obtained by the subtraction method. A positivity as assessed by the subtraction method was actually negative when re-evaluated with fluorescence microscopy (patient 2). In all cases studied, fluorescence microscopy gave results which were consistent with those determined by the HSD method (compare first and last column in Table 3). Discrepancies were obtained with either $2 \%$ preset or $5 \%$ preset backgrounds, respectively (compare columns 2 or 3 with the last column in Table 3). 


\section{Discussion}

The identification of tumour cells in bladder cancer is useful for the detection of tumours as well as for the control of tumour evolution. Specific monoclonal antibodies have been developed which allow tumour antigen identification. However, these antigens, as in other cancer tumours, can be expressed with a high degree of variability (M344, for example). Flow cytometry is an appreciable tool for the pathologist provided the handling of the data is done properly; because of cellular autofluorescence, the main problem is to estimate the proportion of true positive cells for specific antigens in cell populations where these antigens are weakly expressed. In such instances, signal recording methods lead to overlapping representations (in dot plots as well as the corresponding histograms).

As a model study, we analyzed the expression of M344 tumour associated antigen which is expressed in $70 \%$ of superficial bladder tumours [2]. In bladder washings, a convenient way for the follow-up of patients, one often observed an increased non-specific fluorescence background as compared to bladder tumour, and as a consequence an increased overlapping the blank and test data.

We compared several methods of blank evaluation available on commercial software of flow cytometry instruments. The evaluation of the proportion of positive cells was usually independent of the method used if the antibody utilized for investigation was strongly expressed in one cell population. Threshold determination taking into account the shape of the distribution curve (this paper), preset background thresholds, subtraction method or K-S statistics gave identical results in such instances. This was the case for T16 positive urothelial cell identification in tumour samples as well as bladder washings. These results are in agreement with studies analyzing constructed histograms from a known percentage of positive cells; they showed that a $2 \%$ of background method and a subtraction method gave results close to the true values when blank and test fluorescence populations are well separated $[10,12]$.

However, all these methods may lead to erroneous evaluation when the antigen is weakly expressed and/or if the background fluorescent is high relative to that of the positive signal. Our controls by fluorescence microscopy shown that in such instances, the subtraction method and the $\mathrm{K}-\mathrm{S}$ test, and less frequently the $5 \%$ of background method, overestimate the percentages of positive cells and can lead to false positive assessments. They show too that, in relation to higher non-specific fluorescence, the $5 \%$ and $2 \%$ of background methods can underestimate the proportion of positive cells, and thus lead to false negatives. Studies of model histograms have shown similar erroneous results with the preset backgrounds and the subtraction method, when the histograms were overlapping $[10,12,14]$. Finally, the HSD method appeared to be the most reliable method to analyze flow cytometry data; nevertheless, it could lead to false negative estimation in the case of particularly intense background (one case in our study, not shown).

In difficult cases, the fluorescence microscope remains the only reliable tool for positive cell evaluation, since it provides an analysis of the FITC fluorescence texture which is specific in positive cells and may be distinguished from background fluorescence. However, this method is time consuming and requires an experienced cytologist. Thus, it can be applied, as in this report, to control the accuracy of several different analytical methods used to study the expression of a specific antigen in a characteristic tumour population.

Flow-cytometry analysis remains a rapid and useful quantitative method; the method of data analysis may be chosen according to the risk of false positivity or false negativity undertaken by the clinician, in such a way that only a few samples would have to be verified by further microscopic examination. 


\section{Acknowledgments}

We are grateful to J. Albert for flow cytometry measurements, to Drs C. Gorka and T. Rabilloud for helpful discussions. Flow cytometry data presentations were made using ProCyt ${ }^{\circledR}$, a flow cytometry data presentation software developed in our laboratory (available on request). Monoclonal antibodies used in the present study were kindly provided by Dr Y. Fradet.

\section{References}

[1] A.G. Aprikian, A.S. Sarkis, V.E. Reuter, C. Cordon-Cardo and J. Sheinfeld, Biological markers of prognosis in transitional cell carcinoma of the bladder: current concepts, Semin. in Urol. XI(3) (1993), 137-144.

[2] A. Bergeron, S. Champetier, H. LaRue and Y. Fradet, MAUB is a new mucin antigen associated with bladder cancer, J. Biol. Chem. 271 (1996), 6933-6940.

[3] A. Chabanas, J.J. Rambeaud, D. Seigneurin, Y. Fradet, J.J. Lawrence and G. Faure, Flow and image cytometry for DNA analysis in bladder washings: improved concordance by using internal reference for flow, Cytometry 14 (1993), 943-950.

[4] D.K. Chopin and J.C. Laurent, Monoclonal antibodies in bladder cancer cytology, World J. Urol. 9 (1991), 75-78.

[5] P. Dean, Data processing, in: Flow Cytometry and Sorting, M.R. Melamed, T. Lindmo and M.L. Mendelsohn, eds, Wiley-Liss, New York, 1990, pp. 415-443.

[6] Y. Fradet and C. Cordon-Cardo, Critical appraisal of tumour markers in bladder cancer, Semin. in Urol. XI(3) (1993), $145-153$.

[7] Y. Fradet, N. Islam, L. Boucher, C. Parent-Vaugeois and M. Tardif, Polymorphic expression of a human superficial bladder tumour antigen defined by mouse monoclonal antibodies, Proc. Natl. Acad. Sci. 84 (1987), 7227-7231.

[8] Y. Fradet, L. Lafleur and H. La Rue, Strategies of chemoprevention based on antigenic and molecular markers of early and premalignant lesions of the bladder, J. Cell Biochem. (Suppl.) 161 (1992), 85-92.

[9] Y. Fradet, M. Tardif, L. Bourget, J. Robert and the Laval University Urology Group, Clinical cancer progression in urinary bladder tumours evaluated by multiparameter flow cytometry with monoclonal antibodies, Cancer Res. 50 (1990), 432-437.

[10] F. Lampariello, Evaluation of the number of positive cells from flow cytometry immunoassays by mathematical modeling of cellular autofluorescence, Cytometry 15 (1994), 294-301.

[11] L.D. Loar, D. Dennison and S. Sell, Production and characterization of monoclonal antibodies to rabbit lymphocyte subpopulations, J. Immunol. 137 (1986), 2784-2790.

[12] W.R. Overton, Modified histogram subtraction technique for analysis of flow cytometry data, Cytometry 9 (1988), 619-626.

[13] I. Scher, A.K. Berning, S. Kessler and F.D. Finkelman, Development of B lymphocytes in the mouse; studies of the frequency and distribution of surface IgM and $\operatorname{IgD}$ in normal and immune-defective CBA/N F1 mice, J. Immunol. 125(4) 1686-1693.

[14] T.L. Sladek and J.W. Jacobberger, Flow cytometry titration of retroviral expression vectors: comparison of methods for analysis of immunofluorescence histograms derived from cells expressing low antigen levels, Cytometry 14 (1993), 23-31.

[15] I.T. Young, Proof without prejudice: use of the Kolmogorov-Smirnov test for the analysis of histograms from systems and other sources, J. Histochem. Cytochem. 25(7) (1977), 935-941. 


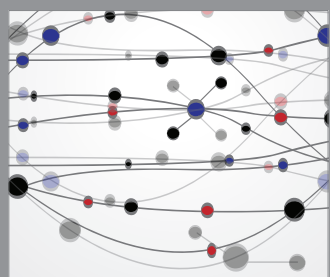

The Scientific World Journal
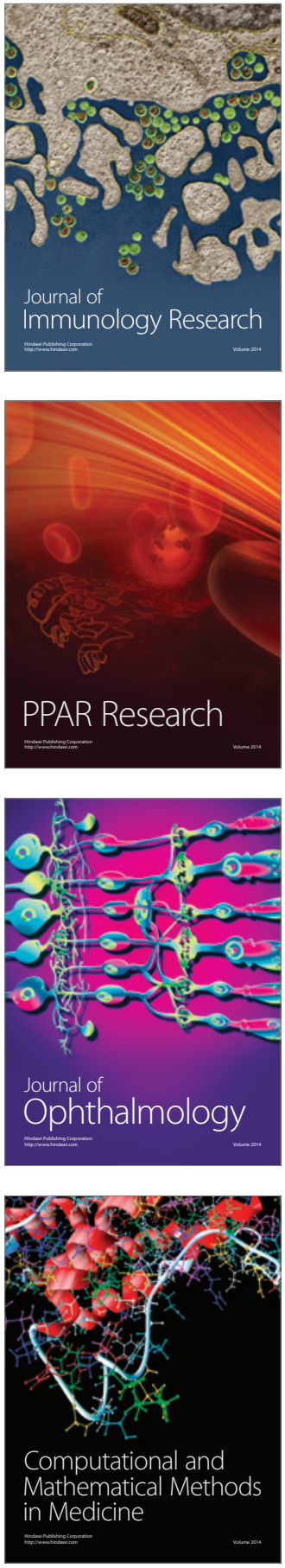

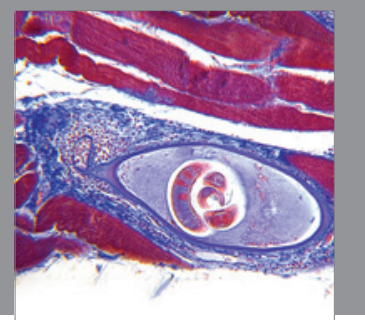

Gastroenterology

Research and Practice
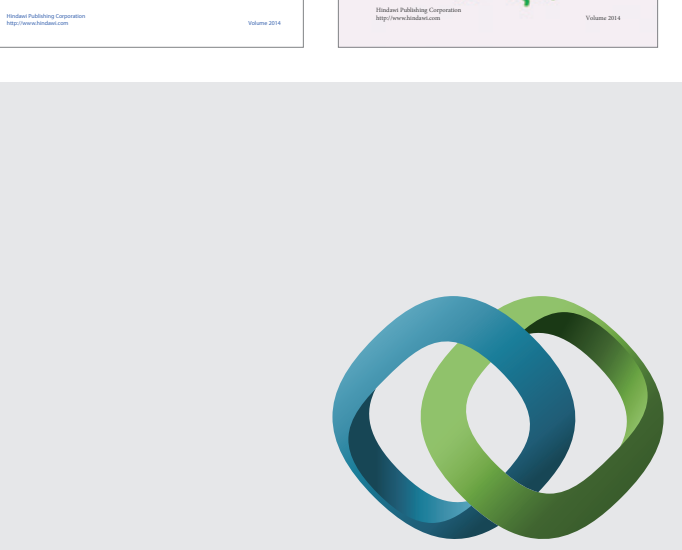

\section{Hindawi}

Submit your manuscripts at

http://www.hindawi.com
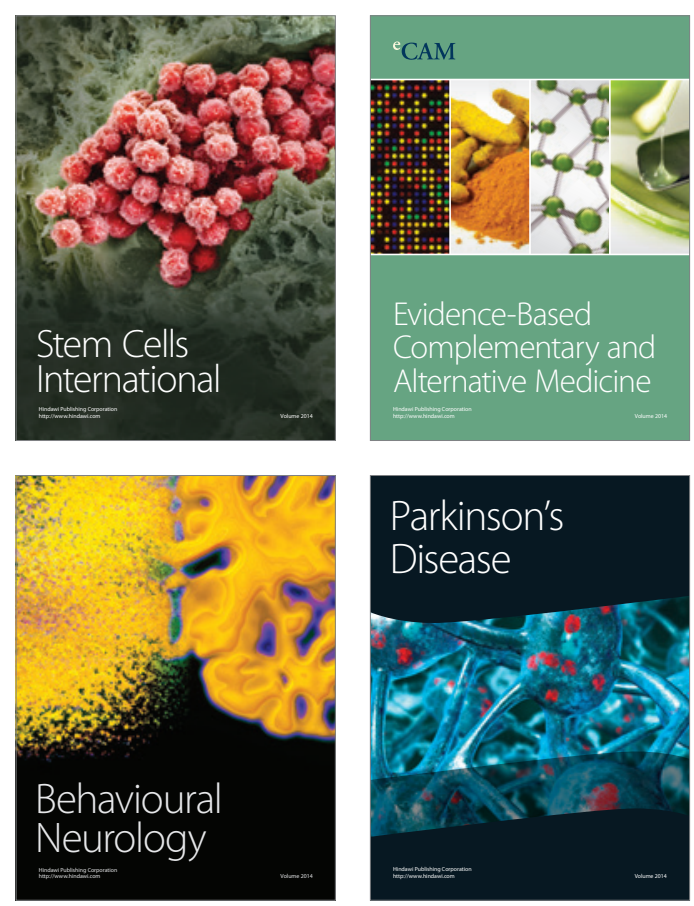

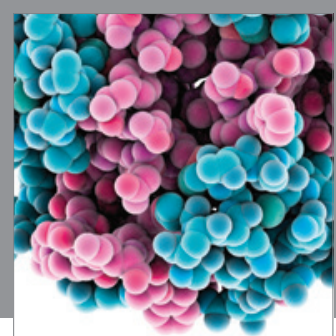

Journal of
Diabetes Research

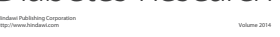

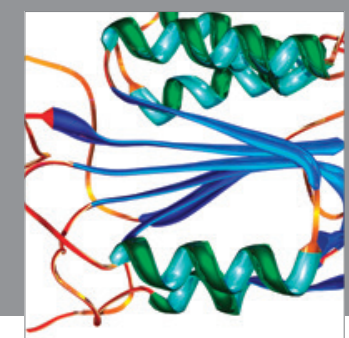

Disease Markers
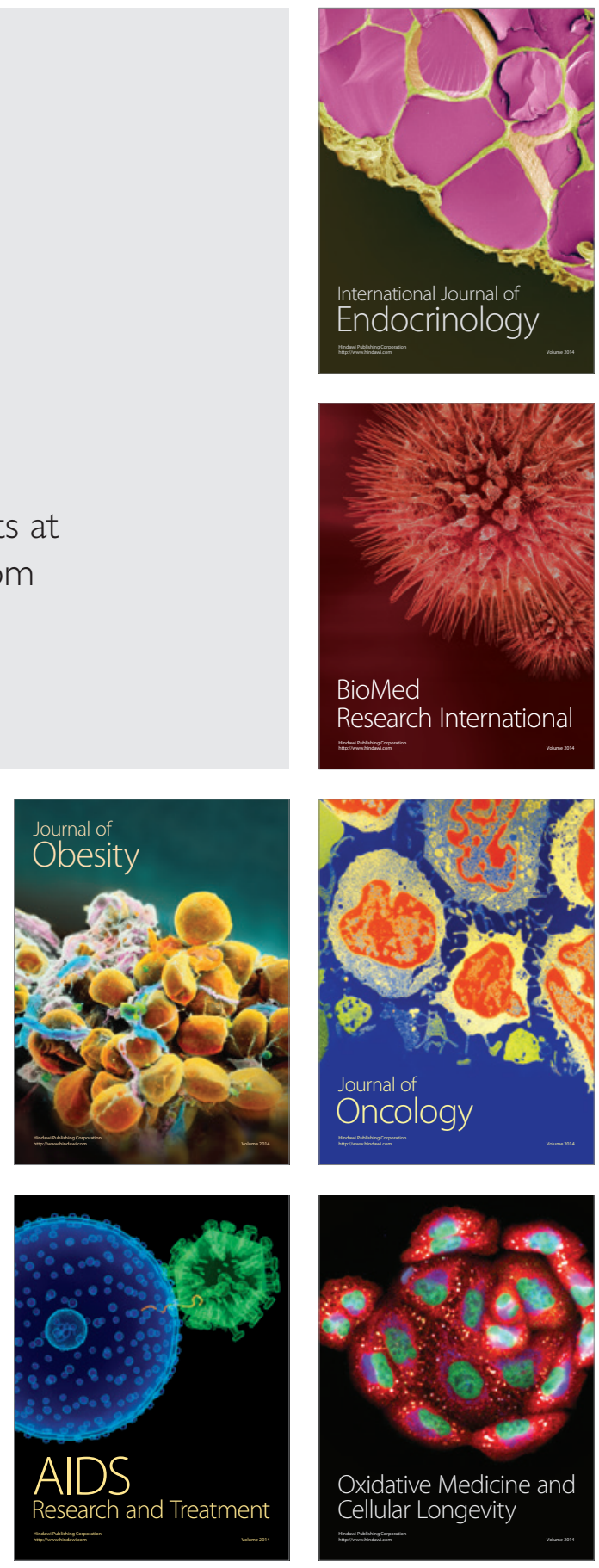